\title{
ECONOMÍA Y CRECIMIENTO POBLACIONAL EN TABASCO
}

\author{
Beatriz Pérez Sánchez*, Ana Bertha Vidal Fócil**, Jesús Alberto Morales Méndez ${ }^{* * *}$, Luis Arturo Méndez Olán***
}

\begin{abstract}
Pérez-Sánchez B., Vidal-Fócil A.B., Morales-Méndez J.A., Méndez-Olán L.A. Economía y crecimiento poblacional en Tabasco. Hitos de Ciencias Económico Administrativas 2014;20 (56): 9-20.
\end{abstract}

\section{RESUMEN}

Objetivo: Investigar el comportamiento de la población a través del estudio de los índices demográficos, entre 1940 y 2010, las políticas de población y su relación con la economía de Tabasco.

Material y método: Con base en los resultados de los Censos de Población y Vivienda del Instituto Nacional de Estadística y Geografía (INEGI), el Programa Nacional de Población 2007-2012 y la información bibliográfica de la economía de Tabasco, se utilizaron los métodos:1) estadístico, 2) histórico, 3) comparativo y 4) analítico. El primero, en la consulta y procesamiento de datos estadísticos; el segundo y tercero dan mayor peso al origen histórico y comparativo para conocer los patrones demográficos; y cuarto, atiende las políticas económicas que explican el cambio en la estructura poblacional del Estado.

Resultados: En el siglo XX, la demografía tabasqueña experimentó cambios transcendentales, observándose al principio del siglo, tasas reducidas de crecimiento, crecientes y elevadas durante los cincuenta años posteriores, y con tendencia decreciente en los años setenta, cuando se comienza a regular a nivel nacional el crecimiento demográfico con la creación del Consejo Nacional de Población (CONAPO) en 1974. Entre 1970 y 1980 la población aumentó en un 23. 6 \%; es decir, pasó de 811, 114 habitantes en 1970 a 1, 062, 961 en 1980, a causa de la migración interestatal ocasionada
Pérez-Sánchez B., Vidal-Fócil A.B., Morales-Méndez J.A., Méndez-Olán L.A. Economía y crecimiento poblacional en Tabasco. Hitos de Ciencias Económico Administrativas 2014;20 (56): 9-20.

\section{ABSTRACT}

Objective: To investigate the behavior of the population through the study of the demographic indicators, between 1940 and 2010, the population policy and its relation to the economy of Tabasco.

Material and method: Based on the results of the Population and Housing Censuses of the National Institute of Statistics and Geography (INEGI), the National Population Program 2007-2012, and bibliographic information for the economy of Tabasco methods were used:1) statistical, 2) historical, 3) comparative and 4) analytical. The first in the query and processing of statistical data. The second and third give greater weight to the historical origins and comparative to know the demographic patterns, and fourth attends the economic policies that explain the change in the population structure of the state.

Results: In the twentieth century, the demography in Tabasco experienced momentous changes, observed at the beginning of the century, reduced rates of growth; growing and high during the fifty years later, and with a decreasing trend in the seventies, when it begins to regulate at the national level population growth with the creation of the National Population Council (CONAPO) in 1974. Between 1970 and 1980 the population increased at a 23 percent, i.e. step of 811,114 inhabitants in 1970 to $1,062,961$ in 1980, because of the interstate migration caused by oil deposits, the

*Doctora en Economía. Profesora-Investigadora. División Académica de Ciencias Económico Administrativas (DACEA). Universidad Juárez Autónoma de Tabasco (UJAT).

** Maestra en Administración. Profesora-Investigadora. DACEA-UJAT.

*** Licenciado en Economía.

****Maestro en Administración. Profesor-Investigador. DACEA-UJAT.

Fecha de recibido: 18 de noviembre de 2013 Fecha de aceptación: 16 de diciembre de 2013. 
por los yacimientos petroleros, el mejoramiento en las vías de comunicación y la integración del estado en el mercado nacional.

Conclusiones: En función de las políticas de población en los últimos cincuenta años, las tasas de crecimiento de Tabasco indican un claro retroceso, en los cincuenta se inició con una tasa de $4.47 \%$ y esta tendencia alcanzó su nivel más bajo en el año 2010 con un $2.77 \%$. Los esfuerzos de las políticas de población se dirigen a impulsar la desaceleración del ritmo de crecimiento y promover la distribución territorial de la población de acuerdo con las potencialidades de desarrollo económico. El Estado a través de la política económica, privilegió las inversiones federales en las presas de Chiapas que se manejaron desde Tabasco, las inversiones de la Alianza para el Progreso que se realizaron en los Planes Chontalpa y BalancánTenosique, la infraestructura carretera del Golfo, los apoyos para la producción pecuaria, coprera y cacaotera y el desarrollo de la industria petrolera. improvement in the way of communication and the integration of the state in the national market.

Conclusions: depending on population policies in the past fifty years, the growth rates of Tabasco indicate a clear step backwards, in the fifties began with a rate of $4.47 \%$ and this trend reached its lowest level in the year 2010 with a $2.77 \%$. The efforts of population policies are directed to promote the slowdown in the pace of growth, and promote the territorial distribution of the population in accordance with the potential for economic development. The State through economic policy privilege the federal investments in the dams of Chiapas that were handled from Tabasco, the investments of the Alliance for Progress made in the Plans and constructed from Balancán Chontalpa-Tenosique, the highway infrastructure of the Gulf, the bearings for livestock production, coprera and cocoa and the development of the oil industry.
Palabrasclave: Población. Economía. Petróleo. Historia. Agricultura. Mortalidad. Natalidad. Migración.
Key words: Population. Economy. Oil. Hhistory. Agriculture.Mortality. Fertility. Migration.

DIRECCIÓN PARA RECIBIR CORRESPONDENCIA: Correo electrónico: betyperez10@hotmail.com

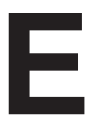

I crecimiento de la población en Tabasco está relacionado con el desarrollo de la economía, la forma particular en que los sectores económicos se desenvuelven no se entiende, si no consideramos las características del Estado mexicano surgido de la revolución que imprimen elementos generales que están presentes en todos los estados y regiones del país, aunque con distintas particularidades dados sus recursos naturales y eventos de corte histórico.

México es un país capitalista desde el último cuarto del siglo pasado, corresponde a la época porfirista (1876-1910) el crecimiento de la economía basado en la explotación de productos agrícolas y de la industria extractiva; es decir, era una economía agroexportadora. El modelo de crecimiento hacia afuera como también se le llamó, privilegió la producción para exportación, descuidando la satisfacción de las necesidades internas que desencadenaron la Revolución Mexicana.
El Estado surgido del conflicto armado de 1910, es considerado capitalista, que tendió a dirigir el proceso económico del país a través de la política económica. La principal expresión del Estado es la Constitución de 1917, la cual convierte al presidente de la república en árbitro supremo del país, encargado de regular las relaciones capitalistas de propiedad y trabajo, básicas para el desarrollo del sistema. El Estado para impulsar el desarrollo se convierte en legislador, empresario público, patrón, inversionista, es decir en agente central de dirección de la economía del país (Méndez, 2012).

En Tabasco, las actividades económicas han estado condicionadas por el medio natural y los mercados. Una serie de etapas caracterizan un producto a lo largo de su historia económica: 1) los cultivos tradicionales, cacao, copra; 2) el monocultivo del plátano (finales del siglo XIX hasta mediados del XX); 3) la expansión ganadera (1950-1970) y la etapa petrolera (1976- 2013). Tabasco es un ejemplo de la teoría clásica del comercio 
internacional fundada en la ventaja comparativa; $\sin$ embargo, la dependencia de un solo producto representa una navaja de doble filo, por un lado el auge y por otro el declive cíclico.

\section{Antecedentes históricos}

El primer hallazgo en la historia de Tabasco se remonta a la llegada de los españoles en 1518 , quienes fundaron su primera ciudad, la villa de San Juan Bautista, hoy Villahermosa. Las villas establecidas se ubicaron generalmente en las riberas de los ríos, Tacotalpa, Teapa, Jalpa, Cunduacán, Jonuta, Nacajuca, Macuspana y Oxolotán ( Gobierno del Estado de Tabasco, 2010). No obstante, a lo largo del periodo colonial, las relaciones con el centro de la Nueva España fueron prácticamente nulas, debido a la lógica mercantilista, primera etapa del capitalismo, que daba mucha importancia a los metales preciosos, siendo Tabasco una región desprovista de recursos minerales como el oro y la plata (Campos \& González, 1983), por ende no había interés en invertir en este territorio, situación que cambia a partir de 1821 con la Independencia.

En México, entre 1900 y 1910, el Producto Interno Bruto (PIB) creció en promedio anual 3.3\% en tanto que la población registró tasas de crecimiento anuales de $1.1 \%$ en el mismo periodo. En términos teóricos se considera que el PIB debe ser mayor que la tasa de población para permitir una mejor distribución del ingreso en la sociedad.

El censo general realizado en 1895 registra en México una población de 12,632,427 habitantes. Los movimientos armados que se iniciaron en 1910 se prolongaron durante varios años, lo que ocasionó una fuerte disminución de la actividad económica, debido a la alta tasa de mortalidad y a la destrucción de bienes de capital y abandono de algunas actividades. La población se redujo de $15,160,369$ en 1910 a 14, 334,780 en 1921. En Tabasco en cambio aumentó de 159,834 a 210,437 habitantes en el mismo periodo. Después de 1920, la actividad económica se reanudó debido al establecimiento de la paz y cierta estabilidad política bajo los mandatos presidenciales de Obregón (1921-1924) y Calles (1925-1928). La tasa de crecimiento de la población fue de $0.1 \%$ anual.

\section{Sector Agropecuario}

En México, a partir de 1930 la economía se empezó a recuperar a diferentes ritmos según la actividad, la agricultura rápidamente, pero la minería y el petróleo a ritmos menores. En la década de 1930 a 1940, el crecimiento promedio anual de la población fue de $1.7 \%$ y el crecimiento económico de $4.48 \%$ anual; estos indicadores permitieron mejores niveles de vida para más mexicanos y sentaron las bases del desarrollo capitalista, se dio un fuerte impulso a la acumulación de capital, así como la creación de infraestructura. En 1930 la población total de México era de 16,552,722 habitantes (CONAPO-INEGI, 1988; INEGI, 1992).

Tabasco es un territorio bañado por los ríos Grijalva y Usumacinta ${ }^{1}$, convirtiéndose así en uno de los más fértiles del país ${ }^{2}$, en consecuencia las actividades agropecuarias son la ventaja competitiva e histórica para la economía tabasqueña. No obstante, la producción se ve realizada hasta que circula y es vendida, de ahí la importancia de las comunicaciones para transportar la producción tabasqueña al interior del país.

En México, el proceso de expansión económica de acuerdo con Solís (1976) se inicia en 1880 con la construcción de ferrocarriles financiados por inversiones extranjeras que también facilitaron el acondicionamiento de los puertos, la explotación de las minas, las operaciones de establecimientos bancarios, transportes y grandes casas de comercio; sin embargo, en Tabasco el incipiente desarrollo de las comunicaciones no permitía que se transportaran las mercancías por ferrocarril, tal como disfrutaban otras regiones del país como el norte y centro, Veracruz, el Istmo y Yucatán desde 1910. Cuando se buscaba entrar a la modernidad, Tabasco apenas contaba menos de 20 kilómetros de vía férrea estrecha, de San Juan Bautista a Carriza, de Cárdenas a Río Grijalva, no concluidas.

Los grandes ejes de los transportes y comunicaciones eran los ríos, donde se concentraba la población; tierra

${ }^{1}$ El volumen medio anual es de 125 mil millones de metros cúbicos, que representan el $35 \%$ de las corrientes del país, formando un amplio cauce que inunda grandes áreas y origina en las zonas bajas numerosas lagunas de poca profundidad. En los meses de septiembre y noviembre se alcanzan los máximos niveles de agua causando inundaciones.

2 Desde los más remotos orígenes, la mayor parte del territorio de Tabasco estuvo cubierta por selva tropical y pantanos; por testimonios, también sabemos de la exuberante riqueza de la flora tabasqueña. 
adentro los únicos medios de transporte eran carros de mulas que transitaban por las brechas precolombinas, de las cuales una fue habilitada como camino real entre San Cristóbal de las Casas y San Juan Bautista. El oro verde (plátano) partía del puerto de Frontera hacia los mercados extranjeros y por ello, se planteó la construcción del ferrocarril ante la fiebre platanera que ofrecía una visión optimista del futuro desarrollo de las plantaciones. El cacao, bien podría seguirse transportando en carros muleros, puesto que la producción era apenas de unos cuantos miles de toneladas. En 1935 se iniciaron los trabajos y penosamente se terminaron en 1950, cuando el auge del plátano había finalizado. Esta vía de comunicación quedó reducida al transporte de maíz y maderas preciosas, a su paso provocó cierto desmonte y la aparición de pequeños poblados como Teapa y Tenosique, hoy municipios.

En este contexto, Tabasco fue gobernado por Tomás Garrido (1931-1934) quien se ocupó del sector primario, creó escuelas orientadas a la enseñanza agropecuaria, se utilizaron nuevos métodos como la agricultura mecánica, el uso de abono químico, y poco a poco fueron sustituidos los viejos equipos agrícolas por unos más modernos y eficientes. De 1940 en adelante, se favoreció la realización de diversas obras encaminadas a mejorar los sistemas de producción en el campo. Se desmontaron grandes cantidades de selvas para crear el "Complejo Agroindustrial de la Chontalpa» mejor conocido como el «Plan Chontalpa», que demandó la construcción de carreteras, canales para riego y más de 20 centros de población.

Para impulsar la economía, en 1948 se terminó la construcción de carreteras que unen los centros de población septentrional a sus estaciones: carretera Cárdenas-Estación Chontalpa y la de VillahermosaTeapa, dando un revés inesperado a la vía férrea. Las plantaciones de cacao y caña de azúcar, así como los ingenios, se establecieron a lo largo de estas carreteras (Revel, 1980). Se dio prioridad a la conexión Villahermosa-Cárdenas, porque forma parte del Circuito del Golfo que constituye el enlace Veracruz-Campeche y dentro del territorio tabasqueño, cruzaba las localidades de Cárdenas, Villahermosa, Macuspana y Emiliano Zapata. Para 1951 esta conexión se había convertido en el enlace principal con el resto del país.
Las vías modernas de comunicación fueron creadas también para desarrollar un movimiento de colonización que había iniciado el ferrocarril, pero en una segunda etapa, desde 1950, la construcción de nuevos caminos organiza y lanza la colonización: la carretera Costera del Golfo, pavimentada por completo en 1960, es el estímulo para la instalación de ranchos ganaderos en el este de Tabasco. La ganadería es un sector que presentó una importante expansión desde el gobierno de Carlos Madrazo (1958-1964). La orientación principal fue la venta de carne bovina, la conversión de un millón de hectáreas agrícolas a ganaderas y la adecuación de los precios a escala internacional.

Entre Villahermosa y Frontera, con la red de carreteras de Paraíso y Frontera a Ciudad del Carmen, las plantaciones de cocoteros cubrieron la costa. A partir de 1955 Comalcalco, empezó a ser un principal productor de cacao, y apoyado por el Banco Nacional de Comercio Exterior se consolidó como un comercializador del producto a nivel internacional (Estañol, 2006). Para impulsar la agricultura, en 1967 se terminó la carretera interior Villahermosa-Champotón, que permitió multiplicar la roturación, las milpas y los pastizales. En 1970 se creó en la zona de los ríos el Plan Balancán-Tenosique, en una extensión de 115,668 hectáreas.

La ganadería tabasqueña se caracteriza actualmente por la implementación de tecnologías de producción, con base en el manejo holístico de los elementos de sustento de la ganadería, con un enfoque agroecológico dando sostenibilidad a la actividad, dando oportunidad al desarrollo de otras actividades agropecuarias en paralelo, haciendo una gran diferencia con el manejo de esta actividad en los ochenta, donde no se le daba interés al mejoramiento genético ni a la asistencia técnica; cabe recalcar que los municipios con mayor actividad ganadera son Balancán, Huimanguillo y Macuspana, los cuales han utilizado la producción de leche para integrarse a la industria quesera. En Teapa la actividad platanera se incrementó en una segunda etapa, fenómeno que propició la instalación de industrias empacadoras y una fábrica de empaques de cartón, además de impulsar el desarrollo del trasporte hacia la sierra tabasqueña.

La silvicultura es una actividad no rentable, debido a que la selva ha sido sacrificada en beneficio de otras actividades y de unas cuentas compañías y personas 
que la explotaron irracionalmente durante años. Para 1982 permanecían activos solo ocho de los diecisiete aserraderos y tres viveros en Centro (Villahermosa), Teapa (Puyacatengo) y Balancán; además de la reforestación de algunas superficies en Tenosique, los cuales hoy en día siguen siendo los municipios dedicados a esta actividad.

A pesar de que Tabasco cuenta con 190 kilómetros de litoral y una gran cantidad de ríos y lagunas, la producción pesquera ha sido relativamente baja, por ello y debido a los problemas de contaminación, la pesca se ha trasladado al cultivo de tilapia y semillas de ostión, los municipios de Teapa, Paraíso y Centro registran la mayor producción; Cárdenas, Emiliano Zapata y Centla continúan participando con un crecimiento menos acelerado debido a que la captura es por pesca tradicional.

\section{Sector Industrial}

Entre 1940 y 1950 en Tabasco, durante la presidencia de Lázaro Cárdenas (1934-1940), se inició la exploración petrolera que dio como resultado el descubrimiento de yacimientos en Comalcalco y Macuspana. El hallazgo de gas en Macuspana impulsó la construcción de una planta de absorción en 1958 y un gasoducto en 1960, y ante la demanda de productos derivados del gas natural, seco y líquido, se construyó otra planta de absorción en 1962 (Gobierno del Estado de Tabasco, 2010). Tabasco se incorporó así plenamente en el desarrollo del país gracias a la explotación petrolera y la construcción de infraestructura carretera, hidro-agrícola e hidroeléctrica en la década de 19501960.

En 1970, Petróleos Mexicanos anunció el descubrimiento de importantes yacimientos petroleros en el municipio de Macuspana, en donde se desarrollaron los campos petroleros «Fortuna Nacional», «Vernet», «Morelos» y «José Colomo». ${ }^{3}$ En consecuencia, se generó un gran flujo de mano de obra rural y de otros estados de la república. Para 1972 se concluyeron las construcciones de los pozos Sitio Grande y Cactus, que señalan el inicio del Boom petrolero. Tabasco se responsabilizó del $23 \%$ de la producción de crudo y del $35 \%$ de la producción de gas; sin embargo, el petróleo a su paso dejaba contaminación y eliminaba fuentes de trabajo de la agricultura, la ganadería y la pesca. Una gran parte de la población vivía en condiciones de pobreza (Villarreal, 2000).

A finales de los años setenta, de acuerdo con Leonardo Curzio (1995) la irrupción del petróleo en un contexto en el que la economía mexicana declinaba, Tabasco se convirtió en una entidad clave: el flujo de los recursos

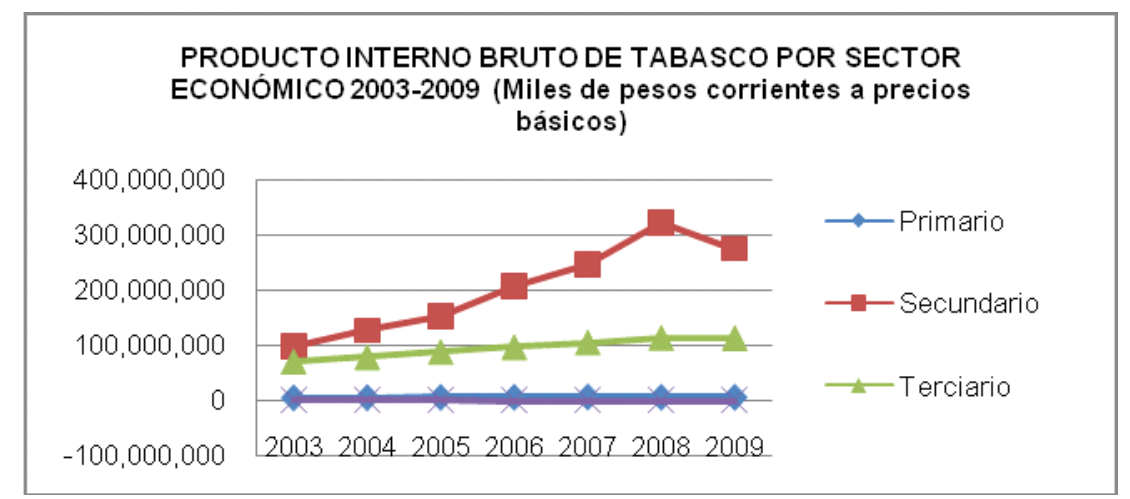

Figura 1. Producto Interno Bruto del Estado de Tabasco por sector económico durante el periodo 2003-2009, expresado en miles de pesos corrientes a precios básicos. Elaborado con base en las estadísticas del INEGI. Página del Instituto Nacional de Estadística y Geografía. «Producto Interno Bruto por Entidad Federativa» Recuperado el 2 de Julio de 2013, de http://www.inegi.org.mx/lib/olap/consulta/General_ver4/ MDXQueryDatos.asp?\#Regreso\&c=17384, 17384

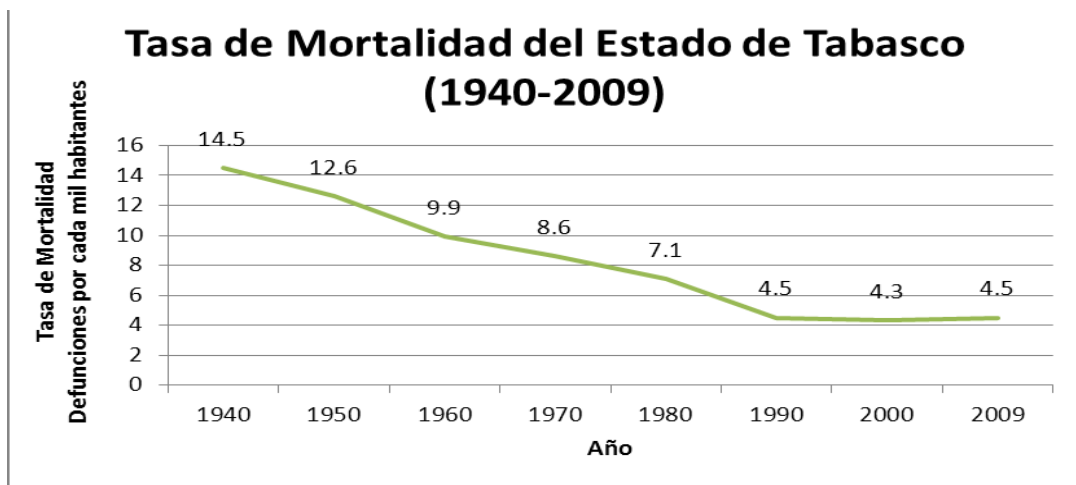

Figura 2. Tasa de mortalidad de Tabasco en el periodo 1940-2009. Elaborado con base en Estadísticas Históricas de México, Gobierno Federal, México, 2009.
${ }^{3}$ El descubrimiento del petróleo en el sureste ocurre en el contexto de la crisis mundial de energéticos y de las presiones de los paí ses miembros de la OPEP por controlar sus riquezas petroleras. 
federales, la migración de trabajadores y la presencia masiva de Petróleos Mexicanos en el Estado son los tres elementos característicos de esta nueva relación Tabasco-México. El aislamiento tradicional de Tabasco se convirtió en una estrecha relación con la capital debido al volumen de participaciones federales.

En 1980 el Estado producía 292,401 millones de barriles de crudo anuales, lo cual representaba el $41.4 \%$ de la producción nacional. En plena crisis de 1982 Tabasco ocupaba el primer lugar en PIB per cápita; no obstante, el $60.9 \%$ de sus habitantes se ubicaba en zonas marginadas (COPLAMAR, 1982).

A partir del inicio del siglo XXI la conformación del PIB se ha ido incrementando en el sector secundario, con una tendencia positiva hasta 2008, dichos incrementos en la producción se explican por la actividad extractiva de naturaleza petrolera que representa la mayor parte del sector. De 2008 a 2009 se ve disminuida la producción, no por decrementos en la productividad sino por la inestabilidad de los precios de los hidrocarburos (consecuencia de la coyuntura financiera surgida en Estados Unidos) ${ }^{4}$.

\section{Políticas e Indicadores Demográficos}

En México, con 19,653,552 habitantes, en la década de los cuarenta se promovieron políticas poblacionales dirigidas a incrementar su número (Gobierno Federal, 1942), así como el ritmo de crecimiento de la población y la densidad demográfica, como requisitos para lograr el desarrollo social y económico. Se instrumentaron acciones tendientes a disminuir la mortalidad que era 22 por cada mil habitantes, conservando el nivel elevado de la natalidad que era de 44.6 (Centro de Estudios Económicos y Demográficos, 1974) y las políticas de migración, para lo cual se promulgó en 1947 la segunda Ley General de Población, al inicio del mandato presidencial de Miguel Alemán Valdés (Kurczyn \& Arenas, 2002), la cual tenía un carácter pro-natalista. La esperanza de vida al nacer era de 41 años.

En Tabasco, en esta década la población era de 285, 630 habitantes (INEGI, 1996), con un moderado crecimiento cuya tasa ${ }^{5}$ era de $2.36 \%$ anual (INEGI, 2000) y escasa población en el territorio estatal con una densidad de 11.3 habitantes por kilómetro

\footnotetext{
${ }^{4}$ El precio del petróleo pasó de 89.43 dólares por barril en 2007 a 41.53 en 2008 (Index Mundi, 2013), dicho comportamiento es explicado por una contracción de la demanda, causada a su vez por la desaceleración económica del periodo.
}

cuadrado. Con referencia a la distribución de la población, $81.98 \%$ residía en áreas rurales del Estado. La tasa de mortalidad $^{6}$ era de 14.5 y de natalidad ${ }^{7}$ era de 39.7 por cada mil habitantes (INEGI, 2009).

En México, como resultado de la aplicación de las políticas de población, en el censo de 1950 la población se estimó en 25.8 millones de habitantes, con un incremento de casi $3 \%$ anual. En Tabasco el censo registró 362,716 habitantes; es decir, se dio un incremento de $3.18 \%$ (INEGI, 2000). La tasa de natalidad era de 43.8 y la tasa de mortalidad de 12.6 .

En el trascurso de la década de los sesenta, se empezó a considerar la posibilidad de crear programas encaminados a regular el crecimiento demográfico. Se realizaron por especialistas una serie de proyecciones sobre la población para el periodo 1960-1980, a nivel nacional y por entidad federativa, que llegaría a convertirse en el fundamento de la programación social y económica del país (Delgado de Cantú, 2003). La población mexicana era de 34.9 millones de habitantes, con un tasa de crecimiento de $3.2 \%$ anual, esta tasa llegó a ser la más elevada del siglo. El índice de natalidad era de 44.9 por cada mil habitantes y el de mortalidad se había logrado disminuir al pasar de 22.8 a 11 por cada mil habitantes y la esperanza de vida al nacer se calculaba en 59 años, elevando 18 años más la esperanza de vida. En Tabasco, en 1960 ya eran 496,340 habitantes, con una tasa de natalidad de $47 \mathrm{y}$ una tasa de mortalidad de 9.9 por cada mil habitantes.

En 1970 la población mexicana fue de 48225 millones y la tasa de crecimiento se calculó en 3.3\% anual. En 1974 se promulgó a nivel nacional la Ley General de Población y se crearon el Consejo Nacional de Población (CONAPO) y el Programa Nacional Indicativo de Investigación Demográfica, del CONACYT. Para esa década la tasa de crecimiento anual en Tabasco era del $4.47 \%$ y la población pasó de 811,114 en 1970 a $1,062,961$ habitantes en 1980. En términos

\footnotetext{
${ }^{5}$ La tasa de crecimiento natural es la diferencia entre nacimientos y defunciones, expresada como porcentaje de la población media, en un determinado periodo.

${ }^{6}$ La tasa bruta de mortalidad representa la frecuencia con que ocurren las defunciones en una población en un determinado año. Se obtiene dividiendo el número de defunciones ocurridas en el año entre la población estimada y multiplicando el resultado por mil, con lo cual se obtiene un tanto por mil de la población total. ${ }^{7}$ La tasa de natalidad se define como el número de nacimientos ocurridos en un periodo, por cada mil habitantes.
} 
demográficos este significativo incremento en la población se explica por el aumento en los niveles de la fecundidad 8 , mientras que los niveles de la mortalidad disminuyeron lentamente. Para esos años, justamente, se desarrollaron nuevas técnicas para la estimación de los niveles de mortalidad; en lo que se refiere a la fecundidad y nupcialidad ${ }^{9}$, se destacaron los avances en el conocimiento sobre el proceso de formación de familias y los tipos de uniones, lo cual modificó en gran medida las tendencias demográficas del Estado para la siguiente década.

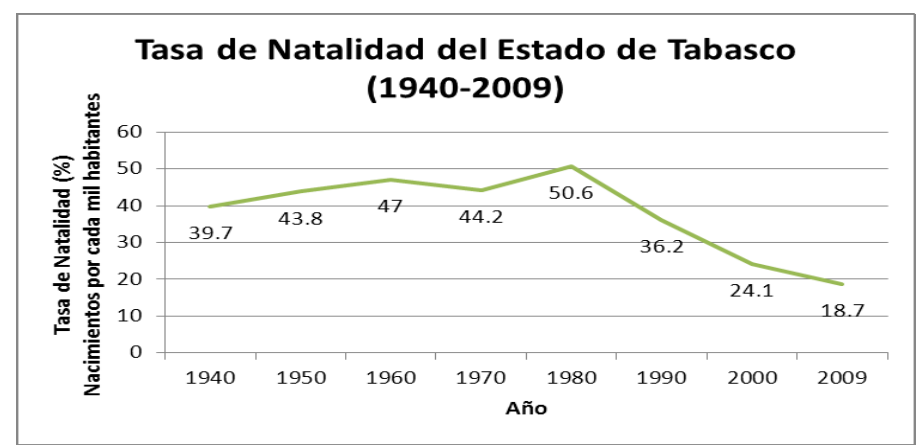

Figura 3. Tasa de natalidad del Estado de Tabasco en el periodo 19402009. Elaborado con base en Estadísticas Históricas de México, Gobierno Federal, México, 2009.

Las actividades económicas han sido las causas del cambio demográfico que ha vivido el Estado; hasta la década de los setenta la distribución de la población se había venido ordenando, en forma equilibrada, en torno a tres zonas principales, en las que se habían desarrollado las actividades económicas fundamentales: la zona oeste, en los municipios de Huimanguillo y Cárdenas; la zona Centro, con los municipios de Centro y Cunduacán; y la zona este, con los municipios de Balancan y Tenosique.

Tabasco registra una superficie territorial de 24661 kilómetros cuadrados. En 2010 se registraron 91 habitantes por kilómetro cuadrado; a partir de 1960, se da un mayor crecimiento en los municipios, cuando se inician la inversiones petroleras en el Estado, se construyen carreteras que lo integran con el resto del país, además del ferrocarril del

${ }^{8}$ La tasa de fecundidad general representa la relación entre los nacimientos vivos y las mujeres en edad fértil (por lo general se consideran aquéllas que tienen de 15 a 49 años). ${ }^{9}$ Número proporcional de nupcias o matrimonios en un tiempo y lugar determinados. sureste, crecen los municipios de Centro, Cárdenas y Huimanguillo, quienes son los receptores de las nuevas inversiones, en 1970 Macuspana crece por la construcción de ciudad PEMEX y la planta criogénica en el mismo lugar. Para 1980 Centro y Cárdenas siguen creciendo, y se anexa Comalcalco a la dinámica de crecimiento.

De acuerdo con las tendencias demográficas, los municipios con mayor cantidad de habitantes son: Centro, Cárdenas, Comalcalco, Huimanguillo, Macuspana y Cunduacán, en conjunto suman; 1,540,475 habitantes en 2010; es decir, el 68.8\% de la población total del Estado, el Centro ha sido y -de acuerdo con las proyecciones del INEGIseguirá siendo el municipio más poblado, por estar asentada la capital del Estado, al 2010 registra 640, 359, habitantes, es decir el $28.6 \%$ del Estado.

Existen cuatro zonas geo-económicas bien diferenciadas: la Chontalpa, poco desarrollada pero con gran concentración de población; la zona de la Sierra, que es la menos poblada del Estado; la de los Ríos, que es una gran llanura aluvial boscosa o selvática; y la del Centro, en la que se ubica la ciudad de Villahermosa, centro de distribución de todos los productos de la entidad, por su importancia geográfica induce al crecimiento de los municipios vecinos de Nacajuca y Jalpa de Méndez. Nacajuca en los últimos 20 años ha incrementado su población en 126\%; pasó de 50,791 a 115,066 habitantes de 1990 a 2010, una explicación es la oferta residencial de ese municipio. Además, se multiplicaron las inversiones petroleras en infraestructura, comunicaciones,

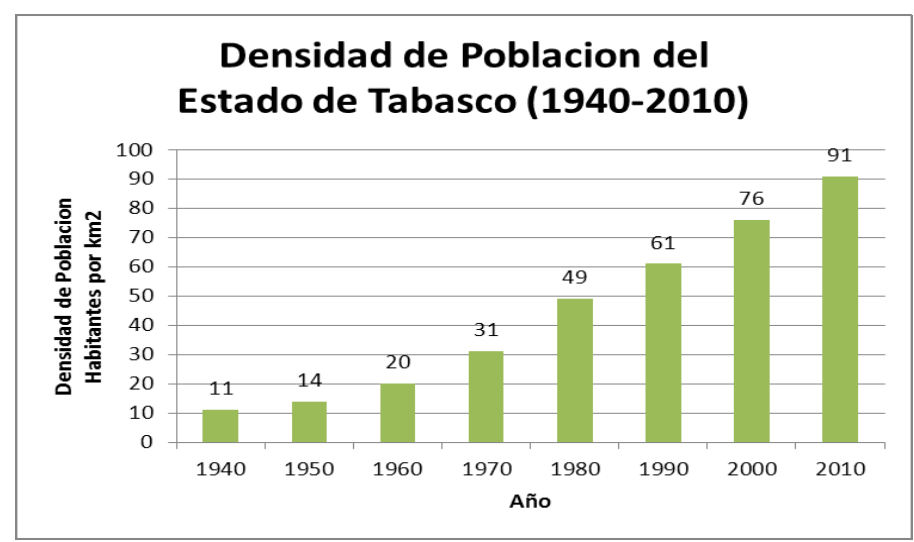

Figura 4. Densidad de Población del Estado de Tabasco (1940-2010). Elaborado con base en las estadísticas del INEGI. Estadísticas Históricas de México, Gobierno Federal, México, 2009. Anuario Estadístico de Tabasco 2010, Gobierno Federal, México, 2010. 
Tabla 1

Población por municipios de Tabasco en orden descendente (No. de habitantes en los años 1990 y 2010)

\begin{tabular}{clcll}
\hline Posición & Municipio & Año 1990 & Municipio & Año 2010 \\
\hline 1 & Centro & 386,776 & Centro & 640,359 \\
2 & Cárdenas & 172,635 & Cárdenas & 248,481 \\
3 & Comalcalco & 141,285 & Comalcalco & 192,802 \\
4 & Huimanguillo & 135,641 & Huimanguillo & 179,285 \\
5 & Macuspana & 105,063 & Macuspana & 153,132 \\
6 & Cunduacán & 85,704 & Cunduacán & 126,416 \\
7 & Centla & 70,053 & Nacajuca & 115,066 \\
8 & Paraíso & 58,403 & Centla & 102,110 \\
9 & Jalpa de Méndez & 57,250 & Paraíso & 86,620 \\
10 & Nacajuca & 50,791 & Jalpa de Méndez & 83,356 \\
11 & Balancán & 49,309 & Tenosique & 58,960 \\
12 & Tenosique & 47,642 & Balancán & 56,739 \\
13 & Teapa & 35,519 & Teapa & 53,555 \\
14 & Tacotalpa & 33,015 & Tacotalpa & 46,302 \\
15 & Jalapa & 28,413 & Jalapa & 36,391 \\
16 & Emiliano Zapata & 22,245 & Emiliano Zapata & 29,518 \\
17 & Jonuta & 22,000 & Jonuta & 29,511 \\
\hline
\end{tabular}

Nota: Elaborado con base en INEGI. Anuario Estadístico del Estado de Tabasco, Gobierno Federal,

México, 1990 y 2010

transportes y servicios gubernamentales, al aumentar las participaciones federales a Tabasco por concepto de explotación petrolera, provocando una concentración poblacional en las ciudades de Villahermosa, Cárdenas y Comalcalco.

Tabasco, comparte con el vecino país de Guatemala, 108 kilómetros de frontera (Aviña Zavala, 2009) que atraviesan la zona selvática del Peten guatemalteco, al mismo tiempo que un puesto fronterizo formal. En el caso de Tabasco, el corredor Naranjo-Ceibo en el Petén guatemalteco, frontera con el municipio de Tenosique, también registra presencia intermitente de asentamientos forestales a principios del siglo XX, y a partir de la década de 1960 experimentó un proceso de poblamiento con personas procedentes de otras regiones de Guatemala que buscaban tierras para actividades agrícolas y ganaderas. Prácticas ilegales como el saqueo de piezas arqueológicas, el abigeato,

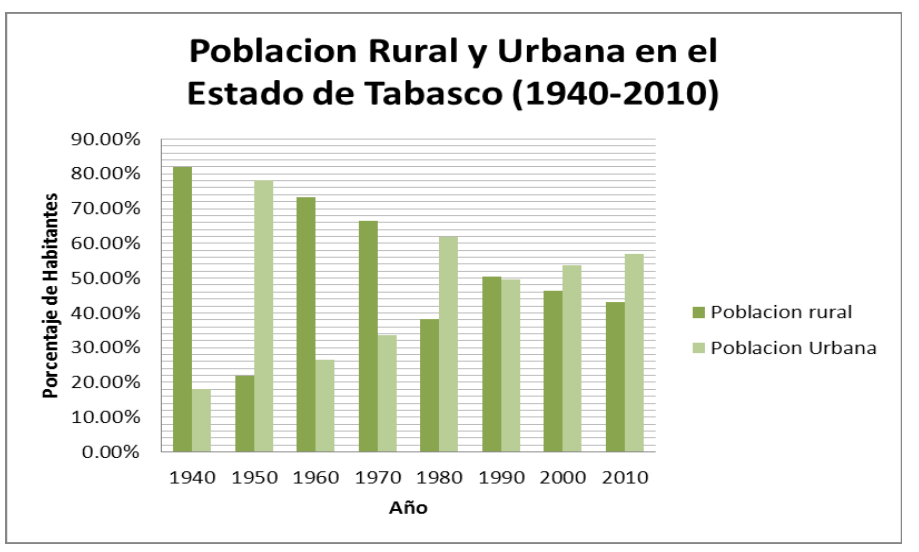

Figura 5. Población rural y urbana en el Estado de Tabasco en el periodo 1940-2010. Elaborado con base en las estadísticas del INEGI. Estadísticas Históricas de México, Gobierno Federal, México, 2009. Anuario Estadístico de Tabasco 2010, Gobierno Federal, México, 2010. 
la caza y comercialización de especies silvestres, así como el contrabando de maderas preciosas, prosperaron en esta región bajo la protección de la selva y la ausencia de controles estatales (Arriola, 2008).

Las guerras civiles en Centroamérica, modificaron los flujos migratorios desde la región hacia y a través de México. A inicios de la década de 1980, ingresaron al país aproximadamente 46 mil guatemaltecos, que más adelante serían considerados como refugiados, los cuales en su mayoría comenzaron a ocuparse en las actividades agrícolas de esta región. Se establecieron primero en el Estado de Chiapas, y en 1984 en Campeche, Quintana Roo y Tabasco. El número de refugiados en los campamentos se había incrementado por el nacimiento de hijos e hijas durante su estancia en dichos Estados, y aunque no se conoce la cifra exacta, algunas fuentes calculan la permanencia de 30 mil ex refugiados en el país (Rolando \& Coello, 2004). De este modo, se puede advertir que la frontera de Tabasco con Guatemala a partir de la década de los sesenta en el siglo pasado, ha tenido consecuencias en el crecimiento de la población de los municipios de los ríos.

\section{Economía y Población}

El reflejo de las actividades económicas se muestra en la Población Económicamente Activa (PEA), con base en el II Conteo de Población y Vivienda 2005 (realizado en 2010). Se define a la población económicamente activa, como aquellas personas de 12 y más años que en la semana de referencia realizaron algún tipo de actividad económica o formaban parte de la población desocupada abierta. En Tabasco,

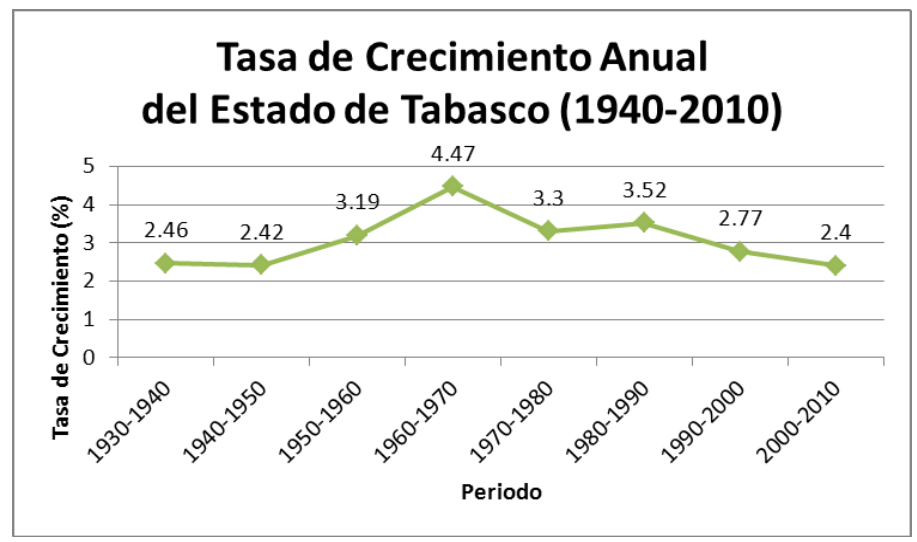

Figura 6. Tasa de crecimiento anual del Estado de Tabasco en el periodo 1940-2010. Elaborado con base en las estadísticas del INEGI. Estadísticas Históricas de México, Gobierno Federal, México, 2009. Anuario Estadístico de Tabasco 2010, Gobierno Federal, México, 2010. son 839,766 de la PEA, y representan el $37.51 \%$ de la población total. La estructura de la PEA por sector registra: $17.95 \%$ primario, $19.39 \%$ secundario y 60.89 terciario (INEGI, 2013). En el sector servicios labora la mayoría de la población.

El sector agropecuario se percibe deficiente y poco productivo, aunque en siglos anteriores la producción del estado se basaba en la agricultura y posteriormente en la ganadería, en la época contemporánea dichas actividades no tienen mayor trascendencia y en su mayoría son desempeñadas sin fin de lucro (autoconsumo). La conducta que traducen tanto el sector primario como el terciario es acorde a la realidad económica a nivel nacional, el caso del secundario es especial, debido a que la inversión como los beneficios son retribuidos de manera indirecta al Estado, a través de participaciones y aportaciones, las cuales solo representan una parte de la productividad.

La tendencia que se ve a nivel estatal en el sector primario, es el reflejo a nivel nacional de un sector insuficiente con dependencia alimentaria (Garrido, 2009), supeditado al comportamiento de la industria petrolera, con una inestabilidad en el precio de las mercancías y con poca retribución para el estado; y un sector de servicios con una postura creciente y estable pero con poca trascendencia y desarrollo en comparación con otras entidades federativas del centro y norte del país. Si no se genera riqueza en los sectores primario y secundario, cómo garantizar el consumo en el sector terciario de manera constante.

El sector secundario o industrial permanece en primer nivel de importancia para Tabasco, si bien su composición se nota inflexible en el corto plazo, la estabilidad del mismo ha permitido el crecimiento del sector III o de servicios, particularmente de las comunicaciones, el comercio y los servicios en general ${ }^{10}$. Con el paso del tiempo en la ciudad de Villahermosa y municipios aledaños se ha venido modernizando la infraestructura adecuada para la distribución de las mercancías, gracias a la inversión pública y privada. Los empresarios que han invertido en Tabasco han preferido el sector servicios

\footnotetext{
${ }^{10}$ La coyuntura de la inundación de 2007 y la crisis financiera de 2008 no han menguado su rendimiento, la primera más grave que la segunda para el estado por el área geográfica en la que se originó.
} 
discriminando la agricultura y la industria debido a la rapidez de la rotación del capital (Ruiz, 2009); el comportamiento de dichos agentes económicos aunque contribuye al PIB estatal, no se compara a la inversión y productividad que el gobierno federal realiza a través de PEMEX. En términos históricos, la agricultura mexicana permitió la acumulación que fue transferida a la industria y los servicios; en contraste, en Tabasco el petróleo le ha permitido modernizarse y promover su crecimiento poblacional pero no ha logrado impulsar la agricultura, fuente de su ventaja comparativa ante la globalización de los mercados.

\section{CONCLUSIONES:}

- Tabasco es resultado de procesos históricos y socioeconómicos endógenos, con una influencia latente de factores exógenos que provocaron su transformación constante y actuaron como vínculo con el exterior; a través del tiempo y del espacio ha conformado actividades productivas que le confieren la categoría de región socioeconómica. Su desarrollo regional se asocia a cuestiones tales como el incremento de la producción y el progreso técnico, la distribución del ingreso, la preservación de los recursos y del medio ambiente en general y la organización territorial que conlleva el crecimiento poblacional.
- La agricultura como medio histórico de producción ha permitido alimentar a la población y entre otras funciones, proveer de materias primas a los sectores industrial $y$ de servicios. El sector rural ha proporcionado en forma abundante, mano de obra barata a estos sectores.

- En Tabasco la decisión del Estado de tomar a la inversión como agente activador del crecimiento económico ha propiciado la explotación de un recurso productivo, el petróleo, con la orientación territorial de los beneficios de las políticas públicas en el renglón de infraestructura y servicios, siguiendo la inercia en cuanto a la generación de actividades agropecuarias para satisfacer la demanda de la población interna, y de exportación. Sin embargo, el petróleo no es renovable y se deberán tomar estrategias productivas alternas. De la acumulación de la riqueza han dependido los indicadores de bienestar tales como bajar la tasa de mortalidad e incrementar la esperanza de vida, la tasa de natalidad constante se ha permitido buscando satisfacer la demanda de población para la producción y consumo futuros.

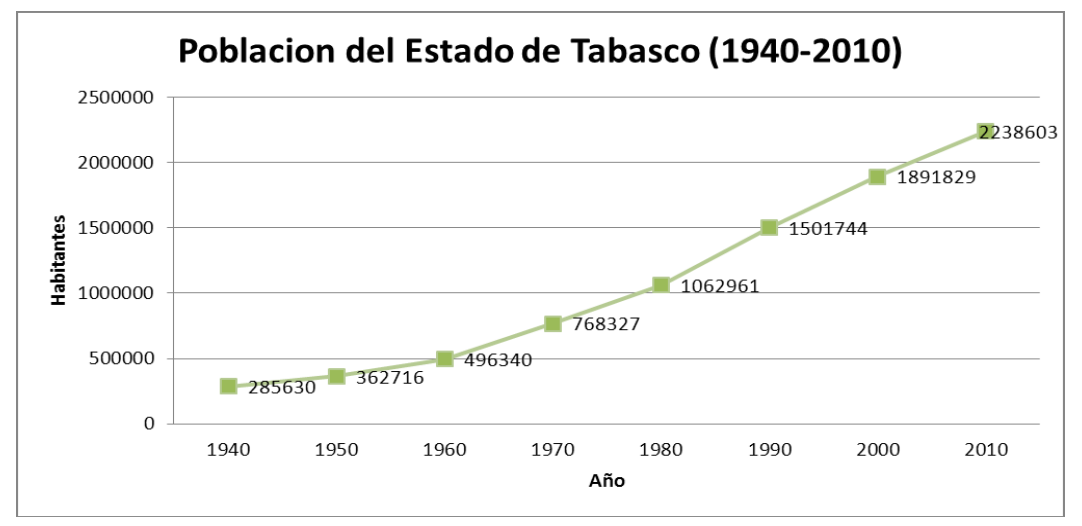

Figura 6. Población en el Estado de Tabasco en el periodo 1940-2010. Elaborado con base en las estadísticas del INEGI. Estadísticas Históricas de México, Gobierno Federal, México, 2009. Anuario Estadístico de Tabasco 2010, Gobierno Federal, México, 2010. 


\section{REFERENCIAS}

Gobierno del Estado de Tabasco. (2010). El Estado de Tabasco: su protagonista. Estampas e Historia. Bicentario de la Independencia. Centenario de la Revolución Mexicana.

Arriola, L. (2008). Frontera-frente y territorialidad en el noroccidente del Petén, Guatemala. (C. Univ. Arturo Prat, Editor). Recuperado de www.revistacienciasociales.cl/archivos/revista21/ pdf/rcs_21.pdf

Aviña, C. (2009). La frontera olvidada: seguridad y migración en la frontera México-Guatemala.

Campos, J., \& González, P. (1983). Tabasco: las voces de la naturaleza. México: Consejo Editorial del Gobierno del Estado de Tabasco.

Centro de Estudios Económicos y Demográficos. (1974). La población de México. México: El Colegio de México.

CONAPO. (1996). Programa Nacional de Población 1995-2010. México.

CONAPO. (1996). Situación Demográfica del Estado de Tabasco. México.

CONAPO-INEGI. (1988). Agenda estadística. México.

COPLAMAR. (1982). Geografía de la marginación. En necesidades esenciales de México,

Situación actual y perspectivas al año 2000 (pág. 64). México: Siglo XXI.

Curzio, L. (1995). Biblioteca de las entidades federativas, Tabasco. México: UNAM.

Delgado de Cantú, M. (2003). México: estructuras política, económica y social. México:

Pearson Educación.

Estañol, E. (2006). Población y crecimiento económico del Estado de Tabasco. Vill a he rmos a, Tabasco.

Garrido, G. (2009). Depende Tabasco en alimentos del país. El Heraldo de Tabasco.

Gobierno Federal. (1942). Sexto censo general de población de los Estados Unidos Mexicanos. México: Dirección de la Economía Nacional, Dirección General de Estadística.

Index Mundi. (2013). index mundi. Recuperado de http://www.indexmundi.com/es/precios-demercado/?mercancia=petroleo-

crudo\&meses $=120$
INEGI. (2013). Recuperado de $\mathrm{h}$ t $\mathrm{t}$ p : / / www.inegi.org.mx/lib/olap/consulta/General_ver4/ MDXQueryDatos.as p?\#Regreso\&c=17384, 17384

INEGI. (1992). Anuario estadístico. México.

INEGI. (1996). Cien años de censos de población. México: Gobierno Federal.

INEGI. (2009). Estadísticas Históricas de México. México: Gobierno Federal.

INEGI. (2013). Instituo Nacional de Estadística y Geografía. Recuperado de http:// www3.inegi.org.mx/sistemas/mexicocifras/

INEGI. (2000). Perfil socio-demográfico Tabasco. México: Gobierno Federal.

INEGI. (2000). Perfil socio-demográfico Tabasco. México: Gobierno Federal.

Kurczyn, P., \& Arenas, C. (2002). La población en México, un enfoque desde la perspectiva del derecho social. México: Instituto de Investigaciones Jurídicas de la UNAM.

Méndez, S. (2012). Problemas económicos de México y sustentabilidad. México: McGraw Hill.

Revel, J. (1980). Aprovechamiento y colonización del trópico húmedo mexicano. España: La vertiente del Golfo y el Caribe, Sección de obras de Economía, F.C.E.

Rolando, D., \& Coello, T. (2004). La decisión de marcharse. Los pueblos indígenas migrantes de Guatemala y Chiapas. (Guatemala, Editor) Recuperado el 2013, de Consejería en Proyectos: www.acnur.org/biblioteca/pdf/5826.pdf

Ruiz, Y. (2 de Diciembre de 2009). Novedades de Tabasco.com.mx.Crece $9.5 \%$ Tabasco en Inversión Privada.

Solís, L. (1976). La realidad económica mexicana: retrovisión y perspectivas. México: Siglo XXI.

Villarreal, D. (2000). La política económica y social de México en la globalización. México:

UAN-Xochimilco. 